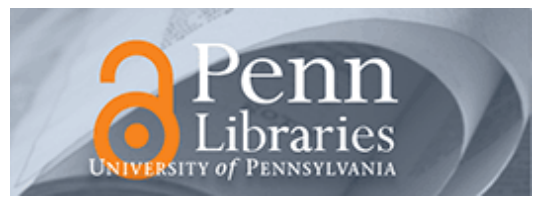

University of Pennsylvania ScholarlyCommons

$12-2003$

\title{
Measuring Imputed Cost in the Semiconductor Equipment Supply Chain
}

Morris A. Cohen

Teck H. Ho

Christian Terwiesch

University of Pennsylvania

Follow this and additional works at: https://repository.upenn.edu/oid_papers

Part of the Operations and Supply Chain Management Commons

\section{Recommended Citation}

Cohen, M. A., Ho, T. H., \& Terwiesch, C. (2003). Measuring Imputed Cost in the Semiconductor Equipment Supply Chain. Management Sciecne, 49 (12), 1653-1670. http://dx.doi.org/10.1287/ mnsc.49.12.1653.25115

This paper is posted at ScholarlyCommons. https://repository.upenn.edu/oid_papers/169

For more information, please contact repository@pobox.upenn.edu. 


\title{
Measuring Imputed Cost in the Semiconductor Equipment Supply Chain
}

\begin{abstract}
We consider the order-fulfillment process of a supplier producing a customized capital good, such as production equipment, commercial aircraft, medical devices, or defense systems. As is common in these industries, prior to receiving a firm purchase order from the customer, the supplier receives a series of shared forecasts, which are called "soft orders." Facing a stochastic internal manufacturing lead time, the supplier must decide at what time to begin the fulfillment of the order. This decision requires a trade-off between starting too early, leading to potential holding or cancellation costs, and starting too late, leading to potential delay costs. We collect detailed data of shared forecasts, actual purchase orders, production lead times, and delivery dates for a supplier-buyer dyad in the semiconductor equipment supply chain. Under the assumption that the supplier acts rationally, optimally balancing the cancellation, holding, and delay costs, we are able to estimate the corresponding imputed cost parameters based on the observed data. Our estimation results reveal that the supplier perceives the cost of cancellation to be about two times higher and the holding costs to be about three times higher than the delay cost. In other words, the supplier is very conservative when commencing the order fulfillment, which undermines the effectiveness of the overall forecast-sharing mechanism.
\end{abstract}

\section{Keywords}

imputed cost, Supply Chain Management, semiconductor equipment

Disciplines

Operations and Supply Chain Management 


\title{
Measuring Imputed Cost in the Semiconductor Equipment Supply Chain
}

\author{
Morris A. Cohen, Teck H. Ho, Justin Z. Ren* Christian Terwiesch* \\ Revision: May 27, 2003
}

\begin{abstract}
We consider the order fulfillment process of a supplier producing a customized capital good, such as production equipment, commercial aircraft, medical devices, or defense systems. As common in these industries, prior to receiving a firm purchase order from the customer, the supplier receives a series of shared forecasts, which are called 'soft orders'. Facing a stochastic internal manufacturing lead-time, the supplier must decide at what time to begin the fulfillment of the order. This decision requires a trade-off between starting too early, leading to potential holding or cancellation costs, and starting too late, leading to potential delay costs. We collect detailed data of shared forecasts, actual purchase orders, production lead-times, and delivery dates for a supplier-buyer dyad in the semiconductor equipment supply chain. Under the assumption that the supplier acts rationally, optimally balancing the cancellation, holding, and delay costs, we are able to estimate the corresponding imputed cost parameters based on the observed data. Our estimation results reveal that the supplier perceives the cost of cancellation to be about two times higher and the holding costs to be about three times higher than the delay cost. In other words, the supplier is very conservative when commencing the order fulfillment, which undermines the effectiveness of the overall forecast sharing mechanism.
\end{abstract}

\footnotetext{
*Operations \& Information Management Dept., the Wharton School, Univ. of Pennsylvania.

${ }^{\dagger}$ Marketing Group, Haas School of Business, University of California-Berkeley.
} 


\section{Introduction $^{1}$}

Many firms selling expensive customized capital goods such as production equipment, commercial aircraft, medical devices, or defense systems, face an order-fulfillment dilemma. On the one hand, their customers expect them to exhibit a high degree of responsiveness, requiring product delivery within an aggressive lead-time. On the other hand, the customized nature of these products makes it risky for suppliers to keep sub-systems or even finished goods in inventory, leading to lengthy and stochastic manufacturing lead-times. To resolve this dilemma, they routinely begin procurement or even production based on forecasted orders, as opposed to waiting for firm purchase orders from their customers. Such forecasted orders, sometimes also referred to as "soft orders", are based on customers' purchase intent as typically revealed to the supplier's marketing and sales function.

There exists, however, a substantial difference between a forecasted (soft) order and a firm purchase order. Responding to changes in their technology and market environments, customers may decide to revise the shared forecasted orders, leading to either changes in purchase quantity, delays in the requested delivery dates, or cancellations of the orders. This raises an interesting question as to how a supplier should respond to the shared forecasts in such a volatile environment. Specifically, the supplier must decide on how to deal with the preliminary nature of a soft order, and - more operationally - define a point in time (a level of information quality) at which to start the corresponding fulfillment process. The supplier can start early, facing the risk of the order not materializing (cancellation cost) or the equipment being finished too early (holding cost), or can delay the starting time until more information becomes available, thereby facing the risk of being late (delay cost). Put differently, the supplier can "Wait and be Late or Rush and be Wrong", neither of which seems desirable.

In this article, we report on an empirical study of forecast sharing related to the ac-

\footnotetext{
${ }^{1}$ We would like to thank the management teams of the supply-chain dyad who generously provided their internal data. We are also grateful for the constructive comments by the departmental editor, the associate editor, and three anonymous referees.
} 
quisition of customized production equipment for the manufacturing of semiconductors. Semiconductor equipment is a prototypical example of the industry characteristics outlined above. Customers, who are responding to the turbulent environment they face in the demand for their end-products, press for short customer lead-times, requiring product delivery within three months or less. At the same time, the complexity and degree of customization of the equipment causes manufacturing lead-times to be long and stochastic, ranging between several months to a whole year.

From an overall 143 soft orders that we collected, 43 were cancelled and thereby never materialized as business for the supplier. From the remaining 100 soft orders, which ultimately were translated into firm purchase orders, another 76 experienced changes with respect to the delivery date. Taking the perspective of a supplier of customized semiconductor production equipment, we develop a formal model addressing the trade-off between an early start of the order fulfillment process (leading to potential cancellation and holding costs) and a delayed start until more information has become available (leading to a potential delay cost).

How this trade-off is resolved depends on the perceived cost structure of the supplier. While traditionally, the supply chain literature has taken these cost parameters as exogenously given and then searched for the optimal operational decision, we take a 'reverse engineering' approach. Based on empirical observation of the supply chain over time, including detailed data of shared forecasts, actual purchase orders, production lead-times, and delivery dates, and on the assumption that the supplier is a rational actor, we reconstruct the cost parameters that explain the observed supply chain behavior. Our estimation results reveal that the supplier perceives the cost of cancellation to be about two times higher and the holding costs to be about three times higher than the delay cost. In other words, the supplier is very conservative when commencing the order fulfillment process, which undermines the effectiveness of the overall forecast sharing mechanism.

Specifically, this paper makes three contributions. First, we formally quantify the effectiveness of an order forecast sharing system in the semiconductor equipment supply chain and show that it is less effective than one would expect. Fearing order cancellation, the 
supplier, by and large, ignores the preliminary forecast information. Second, to the best of our knowledge, this research is, within the field of supply chain management, the first to not only present and analyze a mathematical model, but also to empirically estimate its cost parameters. Thus in contrast to earlier studies, the cost parameters in our research are not exogenously defined, but empirically estimated for the semiconductor equipment supply chain. This novel approach complements the growing literature of supply chain management by introducing an econometric dimension. Our results can be used by buyers who want to infer the cost parameters used by their suppliers in responding to the shared forecast information. Third, we estimate the cost parameters by adopting a 'structural approach' in assuming that the supplier is rational. Consequently, the estimated parameters are robust in face of policy changes in the presence of rational expectations (Lucas, 1976).

The remainder of this article is organized as follows. Section 2 describes the semiconductor equipment industry, including the buyer-supplier relationship under investigation. Section 3 reviews the relevant literature. We present a formal optimization model that captures the essence of the supplier's decision problem in Section 4. Section 5 describes the data and the underlying econometric framework. Section 6 discusses the estimation results. We validate the model in Section 7, and conduct a policy scenario analysis in Section 8. Section 9 concludes the paper.

\section{Semiconductor Equipment Supply Chain}

The demand for semiconductor production equipment is triggered by the demand for the chip supplier's end products, such as micro-processors or memory chips. As illustrated by the recent down-turn in these markets, the demand from PC suppliers exhibits a high degree of uncertainty, leading to wild adjustments in sales forecasts within a relatively short period of time. Market forecasts are done monthly or quarterly at large chip manufacturers for a time period of 2-5 years into the future and are regularly updated following a rolling horizon principle. 
These product-level demand forecasts are translated into capacity requirements and allocated to either existing or new wafer fabs. If the allocated demand is not supported by available capacity, new equipment is ordered. The ordering of the equipment requires chip manufacturers to consider the peak-load capacity requirements and changes in the technical process specifications of new chip generations.

While the translation of product demand into equipment orders seems straightforward, two factors make this computation extremely complex. First, the semiconductor industry is very capital intense and the capital expenditures for new production equipment are the single largest item on a company's earning statement. For example, the industry leader Intel Inc. spent $\$ 5$ billion for equipment acquisitions in the year 2000 alone. Given this magnitude of capital expenditure, even minor under-utilization of equipment can have a dramatic financial impact.

Second, while most semiconductor equipment in the world is operated 24 hours a day for 7 days a week, the actual availability of a specific piece of equipment can be lower. Equipment becomes unavailable as a result of machine break-downs, required qualification procedures, engineering trials, preventive maintenance, etc. Moreover, semiconductor manufacturing is a highly yield-driven process, associated with substantial scrap and the need for rework. While all these factors reduce the average availability, they also introduce substantial variability into capacity planning, aggravating problems resulting from the demand uncertainty.

\subsection{Equipment Acquisition Process}

Once the capacity planning process has generated requests for additional pieces of equipment, an elaborate tool acquisition process commences. This process includes three stages, namely, forecast sharing, manufacturing, and installation.

During the forecast sharing stage, the chip manufacturer (Buyer) creates a forecasted

order (soft order), which is shared with the equipment manufacturer (supplier) via an on-line collaboration system. This soft order includes the tool's specifications, and the 
requested delivery date (RDD). This soft order however is merely preliminary information - opposed to a final purchase order - since in the presence of market and capacity uncertainties, the buyer does not want to commit to an order at such an early stage. The buyer can, after getting more information about his market demand and production yields, decide to (a) cancel the order, (b) to move it to another date, (c) or leave the soft order unchanged.

The supplier becomes aware of the buyer's purchase intent, both through the on-line information system as well as through direct customer interaction from its sales and marketing department. At some point she needs to initiate the production of the tool, which includes procurement of sub-systems from second tier suppliers and the entry of the order into the production schedule ("slotting").

The supplier faces a difficult situation, as starting the order too early can lead to holding and cancellation costs, while starting the order too late can lead to late-shipment costs. The typical manufacturing lead-time of the supplier ranges between 3 and 5 months. The lead-time however exhibits a significant variability as a result of differences in product-mix going through the supplier's facility, changes in equipment demand, process generation, and/or uncertainty in lead-times from the second tier suppliers.

Finally, the tool is shipped to the corresponding fab, where it is installed and must then move through an elaborate qualification process before it can produce commercial output. The overall equipment acquisition cycle is illustrated by Figure 1. In total, the equipment acquisition cycle is approximately one year. Some tools, especially in the lithography domain, can take even longer.

Note that this equipment acquisition process applies only for tools that have already been developed and proven their technical feasibility. The buyer uses a different contract for the supplier during the development of a new piece of production technology. One important difference between such development contracts and the procurement contracts as outlined above is that the buyer might fund more than one supplier for the development of a single tool type. In contrast, once the equipment specification is established, the buyer 
typically switches to a single sourcing approach.

\subsection{Quality of Exchanged Information: The Supply-Chain Dyad}

The authors conducted a joint research project with a major chip manufacturer and one of the largest equipment suppliers. After documenting the overall equipment acquisition process as outlined above, we collected detailed data on the duration of each of the three stages for a total of 100 tool orders.

Among other data, we collected the forecasted tool orders as shared by the buyer with the supplier via the on-line collaboration system over an extended period of time. The buyer provided a quarterly forecast of how many tools he planned to acquire in each of the coming seven quarters. These forecasts were updated following a rolling horizon principle. For example, in quarter 4 of 1998 the buyer forecasted her demand for quarters Q1 1999 to Q3 2000. In quarter 1 of 1999 a certain number of tools were purchased and a new forecasts for quarters Q2 1999 to Q4 2000 were placed.

Figure 2 depicts the forecasts as provided by the buyer in the quarters Q1 1999 to Q1 2001. Each of these shared forecasts is a time series consisting of the seven quarters included in the relevant forecast window. Figure 2 also contrasts the forecasts with the actual tool purchases. We can make two interesting observations. First, the forecasts vary widely, both over time (what is forecasted in e.g. Q1 2000 for the time period of Q2 2000 to Q4 2001) as well as from one forecast to the next (e.g. what is forecasted in e.g. Q2 1999 for Q4 1999 vs. what is forecasted in Q3 1999 for Q4 1999). The former is a consequence of the lifecycle of process generations and the associated need for capacity expansion. The latter is primarily a result of the inherent uncertainty in the industry, especially with respect to the height of the peak demand in the product lifecycle.

Second, we see that - on average - the buyer forecasted for more tool purchases than he ended up ultimately committing to. In other words, there are significantly more soft orders than hard orders, leading to numerous order cancellations. In our dataset, $30 \%$ of the soft orders were cancelled. This reflects the cost structure of the buyer: forecasting too 
few can lead to equipment shortages and potential production losses of the entire wafer fab with a substantial negative financial impact. Forecasting too many however does not directly cost the buyer. This is so because the risk of producing the equipment without having the demand for it is entirely bore by the supplier.

\subsection{Research Goals}

While a systemic inflation of forecasts does not necessarily lead to out-of-pocket costs to the buyer, it can have negative implications on the supplier's perception of the buyer's credibility. This in turn can hurt overall supply chain performance, and thereby - albeit indirectly - the buyer.

The objective of this article is to measure how the semiconductor equipment supplier we studied perceived the cost of cancellation and holding relative to the cost of late shipment. While previous research on coordination and contracting in supply chains has emphasized the importance of forecast sharing and the risks associated with losing credibility, we provide - to our knowledge - the first empirical study to demonstrate these effects econometrically based on actual supply chain behavior opposed to anecdotal evidence. Our results are of direct managerial relevance to the semiconductor equipment supply chain, as they demonstrate that the supplier does not perceive the shared forecasts as credible, which makes the process of forecast sharing by and large ineffective.

\section{$3 \quad$ Related Literature}

Lee, Padmanabhan, and Whang (1997) provide one of the earliest academic discussion of problems related to soft orders and their cancellation. They refer to such orders as "phantom orders", defined as high forecasts of future demand that never materializes, and see them as a key contributor to the bullwhip effect in supply chains.

Problems relating to phantom orders and overly optimistic forecasts have frequently made their way into the business news. For example, Zarley and Damore (Computer Re- 
seller News; May 6, 1996) discuss how PC manufacturers suspected that their customers (distributors) placed phantom orders. As a result, these manufacturers frequently produce only a fraction of the quantity specified in the demand forecast. A comparable situation occurred in the cellular phone industry in the 1994 Christmas season. Motorola experienced significant over-ordering by customers concerned with a potential capacity short-fall (Business Week, March 6, 1995).

Similar problems were experienced at Boeing, which had difficulties in increasing its production of 747s due to parts shortages. Boeing's large supplier base apparently did not trust the company's optimistic demand forecast (indicating a strong growth in 1997) and therefore could not fulfill Boeing's large orders. Only one year later though, following the Asian financial crisis, the supplier's conservatism proved to be a wise decision (Cole, Wall Street Journal, June 26 and September 16, 1997; Biddle, Wall Street Journal, June 10, 1998).

Boeing not only experienced the problem of forecast credibility with their suppliers, but - at the same time - also with their customers. For example, in May 2001, Northwest Airlines cancelled a soft order of 23 Boeing 737 jets, which led Boeing to fall behind Airbus for the first time (Flight International, June 2001).

In addition to this anecdotal evidence on problems related to forecast sharing, the recent academic literature of supply chain management includes several articles providing game theoretical models of this behavior ${ }^{2}$. Closest to our study, Cachon and Lariviere (2001) distinguish between forecast sharing contracts with forced compliance and voluntary compliance. Under forced compliance, the supplier's behavior can be observed and compared to the contracted behavior with a level of detail sufficient for any deviations to be brought up in court. In this case, all market power rests with the buyer and he will therefore design a contract that maximizes his rent derived from the relationship.

Under voluntary compliance, the supplier's behavior is not fully verifiable. This might be a result of some stochastic element in the environment or within the supplier's opera-

\footnotetext{
${ }^{2}$ Chen 2002 provides an excellent review on research in the area of sharing information within a supply chain. Our paper falls into its category of sharing downstream demand forecast information.
} 
tions. In such a situation, courts cannot enforce contract compliance, as the supplier can always argue that she attempted to comply but failed because of some random event.

The situation analyzed in this article is one of voluntary compliance. While there are detailed contracts written between buyer and supplier, they are very hard to enforce. For example, if the supplier is not able to meet the requested delivery date, she could easily find reasons outside her control to explain this. Examples include parts shortages from the second tier supplier, changing delivery dates from the buyer, and/or other external events.

A second stream of research that is relevant to our work relates to the sharing of preliminary information, which is a common practice in product development teams, especially in those proceeding concurrently. Similar to the supplier in our study, who initiates the order fulfillment process prior to receiving a firm purchase order from the customer, development teams frequently begin their work on a new product prior to receiving detailed design specifications from the customer and/or from the market research department.

In this line of research, Krishnan, Eppinger, and Whitney (1997) and Loch and Terwiesch (1998) model the situation faced by a concurrent engineering team where an information receiving development activity must decide on how to rely on the preliminary information provided by the information sender. The information receiver always wants to start early, in an attempt to gain from parallel task execution. Starting early however uses a lower quality of information and thus has a higher likelihood of costly rework. Consequently, the information receiver faces the dilemma "Rush and be Wrong or Wait and be Late" (Terwiesch, Meyer, and Loch 2002). This fundamental trade-off is similar to the supplier's problem as described above and formalized below. A similar tradeoff is also found in the development of new products (see for example, Cohen, Eliashberg, and Ho 1996). 


\section{Model Formulation}

Our objective is to estimate the unobservable costs of cancellation, holding, and delay used by the supplier. We had observed the supply chain dyad, including shared forecasts and final shipments, over an extended period of time. In addition, we had also obtained data about the supplier's internal manufacturing lead-times. Under the assumption that the supplier would behave rationally in balancing the three cost elements, we wanted to impute values for the costs that explained observed supply chain behavior.

The unit of analysis in both our model and the empirical analysis is an order for a single piece of production equipment. The goal of the supplier is to choose a time to commence work on a given order in order to minimize the total costs.

\subsection{The Supplier's Problem}

Consider a time line (see Figure 3) starting at the point in time when the first soft order is received by the supplier $(t=0)$. Associated with this first, preliminary order is a Requested Delivery Date $(R D D)$, which is potentially refined by the buyer over time. At some point in time the uncertainty inherent in the soft order is resolved. Define this point in time as $t=T_{N}$. At $T_{N}$, the tool delivery is requested with a firm delivery date for $t=R D D_{N}$, or the order is cancelled, in which case we define $R D D_{N}$ as infinity.

The supplier faces the following problem when deciding about the time $T_{p}$ at which she begins the fulfillment process on a - potentially soft - order. Specifically, she faces two types of uncertainty, market uncertainty and uncertainty in manufacturing lead-time. Market uncertainty includes the probability that the order is cancelled, which we will label as $p$, as well as any potential changes in the requested delivery date $R D D_{N}$.

From the operations side, the supplier faces uncertainty in the manufacturing lead-time. This may result from traditional lead-time variability in their job-shop like production

environment, changes in product mix or production volume, and/or from variability in delivery lead-times for sub-systems that are ordered from second tier suppliers. 
The supplier must trade-off the cost of beginning too early (cancellation and holding) with the risk of producing too late (delay cost). The problem resembles a traditional newsvendor problem, which occurs in the time domain as opposed to the usual quantity domain. Define cancellation cost, $c$, as the cost incurred by the supplier per unit of time that an order spends in production and later is cancelled. Cancellation costs include the cost for labor and material for an order that the supplier has started to work on. This includes component orders to second tier suppliers that the supplier has to either return (at a charge) or to hold in inventory. Cancellation cost also includes the opportunity cost of not using the capacity for other orders.

Define holding cost, $h$, as the cost incurred by the supplier per unit of time that the tool is produced prior to the date the customer actually needs it, $R D D_{N}$. Holding cost is driven primarily by the capital cost for the expensive equipment, but also includes the cost of storing the equipment. Finally, define delay cost, $g$, as the cost incurred by the supplier per unit of time that the actual delivery date exceeds the $R D D_{N}$, i.e. for the shipment being late. Note that - since our model takes the perspective of the supplier - these delay costs do not capture the delay cost of the buyer, who potentially loses the output of an entire production line as a result of a late shipment. Although the buyer does not face an immediate threat of substitution in the case of late deliveries (due to the single sourcing policy adopted by the buyer), poor delivery performance will impact the supplier's likelihood of receiving contracts for future tool types and technologies.

The various cost components, including cancellation cost, holding cost, and delay cost, are illustrated in Figure 3. While it might be possible to measure holding cost from the cost of capital, machine price, and depreciation, the other two costs (delay cost and cancellation costs) are intangible, and hard to measure directly.

With these cost parameters, we can state the supplier's decision problem as follows:

$$
\begin{aligned}
\operatorname{Min}_{T_{p}} E(\text { Total Cost })= & p c\left(T_{N}-T_{p}\right)^{+} \\
& +(1-p)\left\{h\left[\left(R D D_{N}-L T\right)-T_{p}\right]^{+}+g\left[T_{p}-\left(R D D_{N}-L T\right)\right]^{+}\right\}
\end{aligned}
$$

where $(.)^{+}$denotes $\operatorname{Max}(., 0)$. In the equation above, the first term denotes the expected 
cancellation cost while the second and the third term capture expected holding cost and expected delay cost respectively. We assume that all three costs are linearly increasing with time. This assumption is necessary in order to obtain closed-form solutions that make the estimation easier. The assumption could be relaxed, if the objective were to provide operational support for the supplier concerning the decision of when to begin work on a forecasted order. In such a context, one would have to collect additional data about the detailed shape of the cost accumulation functions. While holding costs indeed are likely to accumulate linearly in practice, the shape of the cancellation cost curve is likely to be lumpy. For example, upon starting on a forecasted order, the supplier is likely to experience an over-proportionally high upfront cost for procurement of components.

Define $S=R D D_{N}-L T$, and let the cumulative distribution for the new random variable be $F(S)$. Moreover, let the distribution for $T_{N}$ be $G($.$) . Equation (1) can be$ rewritten as:

$$
\begin{aligned}
\operatorname{Min}_{T_{p}} E(\text { Total Cost })= & p c \int_{T_{p}}^{\infty}\left(T_{N}-T_{p}\right) d G\left(T_{N}\right) \\
& +(1-p)\left[h \int_{T_{p}}^{\infty}\left(S-T_{p}\right) d F(S)+g \int_{-\infty}^{T_{p}}\left(T_{p}-S\right) d F(S)\right]
\end{aligned}
$$

It is easy to show that $(2)$ is convex in the decision variable $T_{p}$. Thus, there exists a unique cost minimizing starting point $T_{p}^{*}$ at which the supplier should begin production of an order, which is characterized by the solution to the first order condition:

$$
p c G\left(T_{p}^{*}\right)+(1-p)(g+h) F\left(T_{p}^{*}\right)=p c+(1-p) h
$$

Define the expected delay time at the optimal decision as

$$
V\left(T_{p}^{*}\right)=E\left[L T-\left(R D D_{N}-T_{p}^{*}\right)\right]^{+}=\int_{-\infty}^{T_{p}^{*}}\left(T_{p}^{*}-S\right) d F(S) .
$$

It is easy to show that $V\left(T_{p}^{*}\right)$ is non-increasing with the delay cost, non-decreasing with the cancellation cost and non-decreasing with the holding cost, i.e., $\frac{\partial V\left(T_{p}^{*}\right)}{\partial g} \leq 0, \frac{\partial V\left(T_{p}^{*}\right)}{\partial c} \geq 0$, and $\frac{\partial V\left(T_{p}^{*}\right)}{\partial h} \geq 0$. The same is true for $T_{p}^{*}$, i.e., $\frac{\partial T_{p}^{*}}{\partial g} \leq 0, \frac{\partial T_{p}^{*}}{\partial c} \geq 0$, and $\frac{\partial T_{p}^{*}}{\partial h} \geq 0$. 


\subsection{Functional Forms}

In order to empirically reconstruct the cost parameters $c, h$, and $g$, we need to make specific assumptions concerning the underlying distribution function for the arrival time of finalized information, $T_{N}$, and for the distribution function underlying $S$. For the arrival time of finalized ordering information, $T_{N}$, we assume an exponential distribution. Specifically, we assume $G(x)=1-e^{-\alpha x}$. Figure 4 compares the actual data that we collected in the semiconductor equipment supply chain with an exponential distribution where $\alpha=0.21$. A chi-square goodness-of-fit test cannot reject the null hypothesis that the underlying distribution is exponential.

Next, we need to specify a distribution function for $S=R D D_{N}-L T$. Testing separately, we find both $R D D_{N}$ and $L T$ are normally distributed. So a natural assumption would be to assume $S=R D D_{N}-L T$ is normally distributed. However, the normality assumption makes the model less tractable and therefore, in estimation problems similar to ours, a Weibull $(2, \beta)$ distribution is used instead. This distribution is also called the Rayleigh distribution.

In our context it is possible for $R D D_{N}-L T$ to take on negative values, so we shift the Rayleigh distribution to the right by a constant $\delta$. The exact value of this shift $\delta$ will be estimated jointly with the three cost parameters defined above. While the resulting distribution of $S$ no longer is a Weibull distribution, it still has a relatively simple cumulative distribution function, with $F(S)=1-e^{-\beta^{2}(S+\delta)^{2}}$.

Substituting the functional forms into equation (3) we obtain a simplified first order condition:

$$
p c e^{-\alpha T_{p}}+(1-p)(g+h) e^{-\beta^{2}\left(T_{p}+\delta\right)^{2}}=(1-p) g
$$

\subsection{Optimality Condition and Taylor Approximation}

In order to generate a closed form solution, we linearize the objective function in the neighborhood of a target starting date. We show in the Appendix that Taylor approx- 
imation provides a reasonably good approximation and the solution obtained from the approximation is close to the true optimum value. Let the target starting time be $\tau$ around which we expand the exponential function in the above equation (below we set $\tau$ to be 3 months in our estimation). That is, $e^{-\alpha T_{p}}=e^{-\alpha \tau}-\alpha e^{-\alpha \tau}\left(T_{p}-\tau\right)+r_{G}$, and $e^{-\beta^{2}\left(T_{p}+\delta\right)^{2}}=e^{-\beta^{2}(\tau+\delta)^{2}}-2 \beta^{2}(\tau+\delta) e^{-\beta^{2}(\tau+\delta)^{2}}\left(T_{p}-\tau\right)+r_{F}$, where $r_{G}$ and $r_{F}$ are the residual terms for those two expressions. The resulting first order equation is

$$
\begin{gathered}
p c\left[e^{-\alpha \tau}-\alpha e^{-\alpha \tau}\left(T_{p}-\tau\right)+r_{G}\right] \\
+(1-p)(g+h)\left[e^{-\beta^{2}(\tau+\delta)^{2}}-2 \beta^{2}(\tau+\delta) e^{-\beta^{2}(\tau+\delta)^{2}}\left(T_{p}-\tau\right)+r_{F}\right]=(1-p) g
\end{gathered}
$$

For ease of presentation, define $\theta_{1}=p c, \theta_{2}=(1-p)(g+h)$, and $\theta_{3}=(1-p) g$. Note that $g, h$, and $c$, our key parameters of interest, can be fully recovered from $\theta_{1}, \theta_{2}$, and $\theta_{3}$ : $g=\theta_{3} /(1-p) ; h=\left(\theta_{2}-\theta_{3}\right) /(1-p) ; c=\theta_{1} / p$

Re-arranging terms, we obtain the following closed-form solution for $T_{p}^{*}$ :

$$
T_{p}^{*}=\frac{\theta_{1} e^{-\alpha \tau}+\theta_{1} \alpha e^{-\alpha \tau} \tau+\theta_{2} e^{-\beta^{2}(\tau+\delta)^{2}}+2 \theta_{2} \tau \beta^{2}(\tau+\delta) e^{-\beta^{2}(\tau+\delta)^{2}}-\theta_{3}}{\theta_{1} \alpha e^{-\alpha \tau}+2 \theta_{2} \beta^{2}(\tau+\delta) e^{-\beta^{2}(\tau+\delta)^{2}}}
$$

Our approximation approach has a direct analogy in the order fulfillment process of the supplier under study. The supplier has a predefined milestone at which she initiates the manufacturing process. This milestone is then adjusted to reflect order specific considerations, technical tool characteristics, shipment destination, or tool function. This is a natural analogy to Taylor series expansion, where an objective function can be approximated in the neighborhood of a given point, and actions can be adjusted in relation to that given point to improve the objective.

\section{Estimation}

We observed the semiconductor equipment supply chain at the interface of the buyer and the supplier for a total of 18 months, beginning in January 1999. For this time period, we created a complete history of forecast sharing via direct access to the on-line collaboration system described above. The buyer of the supply chain updates the on-line collaboration system on a monthly basis, providing the latest forecasts in the form of soft orders. 
The system enables us to follow every individual equipment order from its initiation as a soft order to the completion of the installation and qualification procedure. For every order, we collected information on its total order lead time (from the time an order enters the system to the time it is fulfilled), the process technology generation that the equipment was designed to support, the function of the equipment, and the destination (geographic location of the fab) to which the equipment is to be delivered. As we collected the data over an extended period of time, we were also able to observe information about order cancellations and requested changes to the delivery date. This database provided an accurate description of forecast sharing between buyer and supplier. The total number of soft orders in the sample was 143, of which 100 were firm orders.

\subsection{Estimation Procedure}

Assume that $\alpha$ and $\beta$, the parameters for $G($.$) and F($.$) respectively, vary across orders and$ that this variation can be explained by a set of independent variables. Let the observations corresponding to the individual pieces of equipment be indexed as $i=1,2, \ldots, I$, and explanatory variables be indexed as $j=1,2, \ldots, J$. Let any given $\alpha_{i}>0$ consist of a "base rate" $\gamma_{0}$, and an order-specific term which can be explained by a set of values of $x_{i j}, j=1, \ldots, J$. In other words, $\alpha_{i}$ can be written as:

$$
\alpha_{i}=\exp \left(\gamma_{0}+\gamma_{1} x_{i 1}+\ldots+\gamma_{J} x_{i J}\right)
$$

The approach of using covariates to endogenize a distribution parameter is common in empirical marketing models as well as operations research models. See Duenyas (1995) for an example of a customer arrival rate which is explained through various attributes of the customer. Similarly we define $\beta_{i}>0$ as:

$$
\beta_{i}=\exp \left(\rho_{0}+\rho_{1} x_{i 1}+\ldots+\rho_{J} x_{i J}\right)
$$

Our notation can be further simplified by labelling the explanatory variables in vector 
form as $X_{i}=\left(1, x_{i 1}, \ldots, x_{i K}\right)^{\prime}$, a $J \times 1$ vector for $\alpha_{i}$ and a $J \times 1$ vector for $\beta_{i}$ :

$$
\begin{aligned}
\alpha_{i} & =\exp \left(\gamma X_{i}\right) \\
\beta_{i} & =\exp \left(\rho X_{i}\right)
\end{aligned}
$$

Where $\gamma$ and $\rho$ are the respective parameters vectors of dimension $1 \times J$ to be estimated.

A complication arises in our empirical estimation. We did not have data on $T_{p, i}$ (the supplier involved in this study did not keep the data). Consequently, we need to estimate the model using finishing time as follows. Denote $F T_{i}$ to be the finishing time of order $i$ (on which we had data), and let $L T_{i}$ be the manufacturing lead time of order $i$ (on which we did not have data). We have the following identity:

$$
F T_{i}=T_{p, i}+L T_{i}
$$

That is, finishing time equals to starting time plus lead time. Let $Y_{i}$ indicate a set of variables that influence manufacturing lead-times. Then we can predict $L T_{i}$ based on the following regression model:

$$
L T_{i}=\eta Y_{i}
$$

where $\eta$ is the parameter vector to be estimated ${ }^{3}$. To control for potential change in capacity utilization over time, we include a dummy variable for each half year in the regression model. We also estimated our model with quarterly and bimonthly dummies, and found the resulting estimates to be robust.

We assume that the finishing time $F T$ is normally distributed.

$$
F T_{i} \sim N\left(\mu_{i}, \sigma^{2}\right)
$$

The normality assumption is common in regression analysis. To validate this assumption, in section 7 (Model Validation) we formally test this assumption, where we find that normality assumption cannot be rejected. Using our previous results, we can predict the expected finishing time $\mu_{i}$ as follows:

\footnotetext{
${ }^{3}$ We fitted quadratic, cubic, and log specifications to our model, but none of them improved the model fit significantly. Therefore, we keep the linear specification in this paper.
} 


$$
\begin{aligned}
\mu_{i}= & \frac{\theta_{1} e^{-\exp \left(\gamma X_{i}\right) \tau}+\theta_{1} \exp \left(\gamma X_{i}\right) e^{-\exp \left(\gamma X_{i}\right) \tau} \tau+\theta_{2} e^{-\exp ^{2}\left(\rho X_{i}\right)(\tau+\delta)^{2}}}{\theta_{1} \exp \left(\gamma X_{i}\right) e^{-\exp \left(\gamma X_{i}\right) \tau}+2 \theta_{2} \exp ^{2}\left(\rho X_{i}\right)(\tau+\delta) e^{-\exp ^{2}\left(\rho X_{i}\right)(\tau+\delta)^{2}}} \\
& +\frac{2 \theta_{2} \tau \exp ^{2}\left(\rho X_{i}\right)(\tau+\delta) e^{-\exp ^{2}\left(\rho X_{i}\right)(\tau+\delta)^{2}}-\theta_{3}}{\theta_{1} \exp \left(\gamma X_{i}\right) e^{-\exp \left(\gamma X_{i}\right) \tau}+2 \theta_{2} \exp ^{2}\left(\rho X_{i}\right)(\tau+\delta) e^{-\exp ^{2}\left(\rho X_{i}\right)(\tau+\delta)^{2}}}+\eta Y_{i}
\end{aligned}
$$

When estimating finishing times, one cannot assume that two subsequent finishing times are independent of each other. Because of potential capacity constraints and / or congestion effects, a longer than average finishing time of the $n$-th order would - everything else equal - increase the likelihood of a longer than average finishing time of the $(n+1)$-st order. We therefore need to consider a joint distribution between $F T_{i}$ and $F T_{i+1}$. Given the normality assumption of the finishing times, FT, discussed above, we use a bivariate normal distribution $(\mathrm{BVN})$ and specify $\left(F T_{i}, F T_{i+1}\right) \sim B V N\left(\mu_{i}, \mu_{i+1}, \sigma^{2}, \sigma^{2}, \lambda\right)$, where $\lambda$ is correlation coefficient. It follows that

$$
\begin{gathered}
\qquad F T_{i+1} \mid F T_{i} \sim N\left(\mu_{i+1}^{\prime}, \sigma^{\prime 2}\right) \\
\text { where } \mu_{i+1}^{\prime}=\mu_{i+1}+\lambda\left(F T_{i}-\mu_{i}\right), \quad \sigma^{\prime 2}=\sigma^{2}\left(1-\lambda^{2}\right)
\end{gathered}
$$

Therefore we can obtain the joint likelihood function:

$$
\begin{aligned}
L= & f\left(F T_{1}, F T_{2}, \ldots F T_{n}\right) \\
= & f\left(F T_{1}\right) f\left(F T_{2} \mid F T_{1}\right) \ldots f\left(F T_{n} \mid F T_{n-1}\right) \\
& \text { where } f\left(F T_{i+1} \mid F T_{i}\right) \text { is the density to }(15)
\end{aligned}
$$

Maximum Likelihood Estimation (MLE) method can now be readily applied to obtain parameter estimates.

\section{Estimation Results}

We use four explanatory variables to predict the dependent lead time variable $L T$ (measured in months): tool generation, tool functionality, tool destination, and forecast changes. 
Tool generation is a binary variable, which is set equal to one if the tool is a newly introduced technology. At the time of our study, one tool type was a new generation and - based on our interviews - we expected this tool generation to have a longer lead-times. Second, some tools in our sample are CMP (Chemical Mechanical Planarization) tools, which are considered premium tools in the industry and our interviews suggested that these tools would have longer lead-times. We define a binary variable PREMIUM that takes on a value of 1 if the tool is of such function, and zero otherwise. Third, we expect tools ordered from and delivered to development fabs to exhibit a shorter lead time. So we include this binary variable DEVFAB to capture this effect. Fourth, our interviews indicated that during the delivery of production tools, changes with respect to RDD were of much higher importance than changes in equipment specifications ${ }^{4}$, therefore we include CHRDD as one key explanatory variable. Extent and frequency of forecast change in our data set are highly correlated (with correlation at about 60\%). They both measure the degree of volatility of forecast changes. For this reason, we only focus on the frequency (CHRDD).

Finally, we control for the potential effect of capacity constraints at the supplier and their impact on manufacturing lead time, by including fixed effects into our model. For example, if capacity was constrained during the first half-year of 2000, then a dummy variable for that half year would pick up the capacity effect in our sample. Note also that the correlation coefficient, $\lambda$, between two successive orders captures potential congestion effects at the supplier's operation.

The explanatory variables for $\alpha$ and $\beta$ are similar to the ones introduced in the regression on LT, namely, process generation (NEW), tool function (PREMIUM), and tool destination (DEVFAB). The correlation matrix of explanatory variables is reported in Table 1.

Like any other empirical study, our model can omit other crucial variables. To partly

\footnotetext{
${ }^{4}$ We did not consider changes in product specification because they are extremely rare in our context. The buyer follows a strategy in which every piece of production equipment has to be exactly the same, thus potential changes in specifications impact both new and existing tools.
} 
address this problem, we have conducted extensive interviews with both buyer and supplier, attempting to identify "qualitative" factors that we have not included in the analysis. One of the authors was on-site for several months, while the other authors had weekly telephone meetings and had made several visits to various factory locations involved in this research. We observed that several crucial variables that could influence lead time such as leadership in buyer and supplier organizations, task division within both organizations, and the relationship between the dyad were kept constant over the course of our study.

As MLE is a nonlinear method, results can sometimes be sensitive to starting points ${ }^{5}$. In order to ensure the parameter estimates are not only locally optimal, we explored the parameter space by testing various sets of starting values. Specifically, we randomly generate 12000 sets of starting values with each of the parameters drawn from a normal distribution with mean 0 and standard deviation 3 , which covers the typical range of the parameter values from our observation. $\theta_{3}$ is fixed at 0.700 (or equivalently $g=1.000$ ) for identification. We also restrict $g, h$ and $c$ to be non-negative. Table 2 reports the parameter estimates that yielded the globally maximum likelihood.

Converting estimated values for $\theta_{1}, \theta_{2}$, and $\theta_{3}$ into cost parameters $g, h$ and $c$, we obtain the following values: $g=1.000, h=3.031, c=2.108$. These results suggest that the manufacturer weighs the holding and cancellation costs about three and two times higher than the cost of delay.

In order to test the effect of explanatory variables on the model, we test the following sequence of nested models:

1. Tool Destination: DEVFAB. The corresponding hypothesis is $H_{0,1}: \gamma_{2}=0$, and $\rho_{2}=0$.

2. Tool Process Generation: NEW. The corresponding hypothesis is $H_{0,2}: \gamma_{3}=0$, and $\rho_{3}=0$.

3. Tool type: PREMIUM. The corresponding hypothesis is $H_{0,3}: \gamma_{4}=0$, and $\rho_{4}=0$.

\footnotetext{
${ }^{5}$ We use GAUSS, a matrix programming language, to perform the estimation task. The algorithm for maximum likelihood estimation is BFGS.
} 
The results for those hypotheses tests are reported in Table 3. Likelihood ratio tests show that each of the hypotheses is rejected (see $\chi^{2}$ test statistic in each table). Thus, all the variables have indeed predictive power in explaining lead-time.

The parameter values of the dummy variables associated with time show that the capacity constraint does vary over time. The first half year of 1999 appears to have the most constrained capacity. However the maximal difference in lead time across these fixed effects is 0.7 month (the average manufacturing lead time is 4.5 months). So we conclude that the capacity constraint affects the lead time but not substantially. This is also consistent with the modest estimate on the correlation coefficient of finishing time. In-depth interviews with the managers at the supplier provide a plausible explanation. Apparently, orders from the buyer always receive a priority in production scheduling because he is the largest customer of the supplier. In fact, a special unit of marketing support was set up within the supplier organization specifically to facilitate the order fulfillment process of the buyer.

\section{Model Validation}

We first validate our normality assumption. We perform both Kolmogorov-Smirnov Goodnessof-fit test and the Chi-square test as described in Law and Kelton (1991). In both cases, the normality hypothesis could not be rejected. We also draw Q-Q plot and distribution plot of residuals (Figure 5), providing also a graphical support for the normality assumption.

To further validate our model, we compare the finish time $\widehat{F T}$ as predicted by our model, with actual finish time FT. Based on the estimates $\widehat{\theta}_{1}, \widehat{\theta}_{2}, \widehat{\theta}_{3}, \widehat{\gamma}, \widehat{\rho}, \widehat{\delta}$, and $\widehat{\eta}$, we 
obtain a predicted value of $F T$ :

$$
\begin{aligned}
\widehat{F T}_{i}= & \widehat{T}_{p_{i}}+\widehat{L T} \\
= & \frac{\widehat{\theta}_{1} e^{-\exp \left(\widehat{\gamma} X_{i}\right) \tau}+\widehat{\theta}_{1} \exp \left(\widehat{\gamma} X_{i}\right) e^{-\exp \left(\widehat{\gamma} X_{i}\right) \tau} \tau+\widehat{\theta}_{2} e^{-\exp ^{2}\left(\widehat{\rho} X_{i}\right)(\tau+\widehat{\delta})^{2}}}{\widehat{\theta}_{1} \exp \left(\widehat{\gamma} X_{i}\right) e^{-\exp \left(\widehat{\gamma} X_{i}\right) \tau}+2 \widehat{\theta}_{2} \exp ^{2}\left(\widehat{\rho} X_{i}\right)^{2}(\tau+\widehat{\delta}) e^{-\exp ^{2}\left(\widehat{\rho} X_{i}\right)(\tau+\widehat{\delta})^{2}}} \\
& \frac{+2 \widehat{\theta}_{2} \tau \exp ^{2}\left(\widehat{\rho} X_{i}\right)(\tau+\widehat{\delta}) e^{-\exp ^{2}\left(\widehat{\rho} X_{i}\right)(\tau+\widehat{\delta})^{2}}-\widehat{\theta}_{3}}{\widehat{\theta}_{1} \exp \left(\widehat{\gamma} X_{i}\right) e^{-\exp \left(\widehat{\gamma} X_{i}\right) \tau}+2 \widehat{\theta}_{2} \exp ^{2}\left(\widehat{\rho} X_{i}\right)(\tau+\widehat{\delta}) e^{-\exp ^{2}\left(\widehat{\rho} X_{i}\right)(\tau+\widehat{\delta})^{2}}}+\widehat{\eta} Y_{i}
\end{aligned}
$$

Figure 6 compares actual values $F T$ and predicted values $\widehat{F T}$. The graph shows an overall good fit with no apparent systemic deviation from the identity line. An Ordinary Least Square (OLS) regression of FT against $\widehat{F T}$ confirms this observation:

$$
\begin{gathered}
F T=0.000+0.999 * \widehat{F T} \\
*: \text { Significant at } 1 \% \text { level } \\
\mathrm{R}^{2}=40 \% ; \text { Number of observations }=100 . \text { F-statistic: } 137.97 .
\end{gathered}
$$

The results show an intercept that is statistically not different from zero ( $\mathrm{p}$ value $=1.00$ ), and a positive and significant slope $(\mathrm{p}$ value $=0.00)$. We conduct a test to check whether the slope is different from 1 , and we cannot reject the hypothesis that slope is one $(\mathrm{p}$ value $=0.63$ ). We also use White's General Test (p.550 in Greene, 1993) to test heteroskedasticity, and could not reject the hypothesis that residuals are homoskedastic.

\section{Policy Scenario Simulation}

Our mathematical model outlined in Section 4, combined with the parameter estimates for cancellation cost, holding cost, and delay cost, enable us to analyze how modifications of cost parameters would impact the supplier's expected delivery performance. Note that the following analysis has been performed based on the cost parameters we obtained for the supply chain dyad underlying the present research study. While this methodology is generalizable beyond this setting, the magnitude of the following effects are likely to vary across different supply chain settings. 
The buyer in our study was interested in the question of what would be the impact of a financial late shipment fee on the timeliness of deliveries. Economic intuition suggests that such penalty would increase the late shipment cost for the supplier, thereby encouraging her to commence production earlier (smaller $T_{p}^{*}$ ). However, we can go one step further than this. Based on our analytical results and the empirical data, we can recompute the expected shipping delay with any given late shipment cost parameter. This is depicted by Figure 7a, which shows the relationship between the cost parameter $g$ and the expected slippage, as defined in Equation (4), averaged over all orders and expressed in month. Currently, $g=1$ and the corresponding slippage is a little less than 0.4 month. Increasing late shipment cost from $g=1$ to $g=2$ translates into a .15 month reduction in late shipment.

Next, consider the impact of holding cost $h$ on the expected shipping delay (Figure 7b). Again, the status quo corresponds to $h=3$ and a delay of .34 months. Now consider what happens if holding costs are cut in half. For example, the buyer could accept the equipment prior to the specified requested dock date (and of course, also pay for it earlier). Alternatively, the buyer could partially reimburse the supplier for the holding costs. Reducing holding costs from 3 to 1 would lead to a .1 month reduction in shipping delay. While, obviously the financial burden of capital cost now rest with the buyer (who pays for the equipment and then leaves it idle up to the time of actual need), the buyer might still be better off, as shipment delays can put the production of entire fabs at risk, thereby having a much larger impact on the bottom line.

Similarly, Figure 7c and 7d investigate the relationship between changes in cancellation cost $c$ and cancellation probability $p$. A reduction in cancellation cost could be achieved, if the buyer would take over some of the cost incurred by the seller in the case of cancellation (e.g., procurement cost). Alternatively, the buyer could develop specifications with more standardized components in it, which would allow the supplier to reuse entire sub-assemblies for another customer after receiving notice of cancellation.

Interestingly, none of the cost changes outlined above is able to eliminate the expected delay. The reason for this lies in the complex trade-off that the supplier faces when 
deciding upon the optimal time to start working on an order $T_{p}^{*}$. This trade-off not only involves two forces, but rests on a subtle balance between three forces. Hence, even a large improvement along one dimension will only lead to a small change in the supplier's decision - and thereby the expected delay - as the other two forces are still unchanged. Consequently, substantial changes in expected delay can only be achieved by changing at least two of the cost parameters jointly (opposed to changing them one at a time). This is illustrated in Figure 8. We see that reductions in holding cost and in cancellation costs actually complement each other, opposed to acting as substitutes. A $50 \%$ reduction in both of them $(h=1.5, c=1)$ would reduce the expected delay by .15 months, while the two changes implemented individually would lead to a lower reduction of the delay (.1 months for the reduction in $h$ and .03 for the reduction in $c)$. That is, the joint changes are super-additive.

\section{Discussion and Conclusion}

Our results indicate that the supplier fears holding costs and order cancellations, making her averse to commencing order fulfillment based on soft orders. This results from the fact that the supplier's effort, including procurement of components and the actual building of the equipment, is very customer specific. We also find that the supplier perceives holding cost and cancellation cost as much more important relative to the cost of delay.

The large emphasis on early completion cost relative to late completion cost clearly does not mirror the overall cost for the supply chain. If the tool is finished early, it remains at the supplier's plant and only traditional inventory holding costs are incurred. However, a late shipment of the tool can lead to idle time and lost output at the buyer's fab, which is associated with substantial margin losses which are magnitudes larger than the holding cost for a piece of equipment. This suggests there is a lack of coordination in the supply chain which can lead to sub-optimal performance.

The current situation can be partly explained by the fact that the customer in this study is a dominant player in semiconductor products, and is the single largest customer 
of the supplier. This creates an imbalance of power in the buyer-supplier relationship, which is why the customer can provide forecasts without commitment, and can change order forecasts without penalty, leaving the supplier bear all the resulting costs.

The overall supply chain performance could be improved if the customer would be willing to share some of the holding cost. One operational way of doing this would be if the customer accepted the tool delivery for some time window prior to the RDD. This would reduce the expected holding cost for the supplier and thereby move the optimal starting point $T_{p}^{*}$ forward in time. In general, given the high degree of customization demanded by the buyer, supply chain performance also could potentially be improved if the buyer would be ready to share some of the risk of cancellation. This would have two beneficial effects. First, it would reduce the supplier's cancellation cost, moving the optimal starting point $T_{p}^{*}$ forward in time. Second, it would make the forecast more credible, and thereby rebuild some of the trust missing in the system. In presence of a cancellation fee, phantom orders become costly to the buyer, allowing the supplier to have more confidence in the soft orders. Currently, forecasts are provided by the individual fabs of the customer. While all fabs are part of the same company, they also have local objectives. In such a setting, those fabs do not incur the full negative externalities that cancellation has on the reputation of the company as a whole. This suggests that a better coordination of forecasting activities across fabs could be beneficial.

The sharing of demand forecast information does not have a positive value for the supplier, who distrusts these data and delays the production start for the equipment. In our situation of voluntary compliance (the buyer cannot monitor the supplier), financial incentives are needed to make signals related to the forecast credible (Cachon and Lariviere 2001). As the buyer, who is in control over the design of the coordinating mechanisms in the supply chain, does not incur any financial loss in case of a cancellation, such credible signaling is not possible. This would change if the buyer would pay some cancellation fee (potentially as a function of time). While having the right of free cancellation obviously is attractive to the buyer and potentially saves him some direct out-of-pocket cost, the buyer pays a (much higher) price indirectly, resulting from shipment delays and long tool 
delivery lead-times generated by the supplier's response to the current system structure.

We believe that these results are of substantial interest, both from an academic as well as from a direct managerial perspective. On the academic side, these results provide the first econometric evidence of problems related to forecast sharing. While there is a rapidly growing stream of research following Lee et al. (1997), no previous study could econometrically demonstrate the existence of the coordination problems. While the magnitude of the parameter estimates we report in this study is specific to our research setting, our method can easily be implemented to obtain estimates in other supply chain dyads.

From a managerial perspective, our results demonstrate that information sharing by itself is not sufficient to build superior supply chain performance. Our results were presented to senior executives at both the customer and the supplier. In response to our study, the customer started several projects with the objective to overcome some of the credibility problems. For example, one project that the customer initiated attempts to acknowledge the uncertainty inherent in forecasts by communicating it explicitly via a range of possible orders (an interval) as opposed to ignoring it (simply sharing point-based forecasts). In the context of product development, such set-based information exchange has been documented to lead to improved performance.

Finally, we believe that our work not only serves as the empirical foundation for much of the contracting research, but that it also provides a fruitful starting point for future research. A larger empirical study could analyze how the cost parameters that we estimated change over time. For example, one would expect that the cancellation of an order directly increased the supplier's perception of cancellation cost in the subsequent period. Another interesting research opportunity relates to how the forecast is shared. Similar to the field of concurrent product development discussed in the literature review, where there has been a recent trend towards set-based - opposed to point-based - information exchange, the buyer could provide multiple scenarios of demand to the supplier or could even share a confidence interval. This would be consistent with established supply chain concepts such as minimum purchase commitments and its effect on forecast credibility would be interesting to study both analytically as well as empirically. 


\section{References}

Cachon, G. P., Lariviere, M. A., "Contracting to assure supply: How to share demand forecasts in a supply chain", Management Science 47 (5), 629-646, May 2001.

Chen, F., "Information Sharing and Supply Chain Coordination", To appear in the Handbook of Operations Research and Management Science: Supply Chain Management, by T. de Kok and S. Graves (eds.), North-Holland.

Cohen, M., J. Eliashberg, and T-H, Ho, "New Product Development: The Performance and Time-to-Market Tradeoff," Management Science, 42 (1996), 173-186.

Duenyas, I., "Single Facility Due Date Setting with Multiple Customer Classes", Management Science 41(4), 608-619, April 1995.

Greene, W., Econometrics Analysis, NY, Macmillan Publishing Company, 1993.

Krishnan, V., S. D. Eppinger, D. E. Whitney, "A Model-Based Framework to Overlap Product Development Activities", Management Science, 43(4), 437-451, April 1997.

Law, A.M., and Kelton, D.W., 2000, Simulation Modeling and Analysis, McGraw-Hill, Inc.

Lee, H. L., Padmanabhan, V., Whang, S. J., "Information distortion in a supply chain: The bullwhip effect", Management Science 43 (4): 546-558, April 1997.

Loch, C. H., C. Terwiesch, "Communication and Uncertainty in Concurrent Engineering", Management Science, 44(8), August 1998.

Lucas, R. E. "Econometric Policy Evaluation: A Critique" in Bruneer, K. and A. H. Metzler (eds.), The Phillips Curve and Labor Markets, North-Holland, Amsterdam, 1976.

Terwiesch, C, A. De Meyer, C. H. Loch, "Exchanging Preliminary Information in Concurrent Engineering: Alternative Coordination Strategies", Organization Science, 13(4), 402-419, 2002.

\section{Appendix: Taylor Expansion}

The function we want to approximate is Equation (5), the first order condition for the cost minimization problem. 


$$
p c e^{-\alpha T_{p}}+(1-p)(g+h) e^{-\beta^{2}\left(T_{p}+\delta\right)^{2}}=(1-p) g
$$

As before, denote:

$$
\left\{\begin{array}{ccc}
\theta_{1} & = & p c \\
\theta_{2} & = & (1-p)(g+h) \\
\theta_{3} & = & (1-p) g
\end{array}\right.
$$

We can rewrite the first order condition in the form of $f(x)=0$, i.e.,

$$
f(x)=\theta_{1} e^{-\alpha T_{p}}+\theta_{2} e^{-\beta^{2}\left(T_{p}+\delta\right)^{2}}-\theta_{3}=0
$$

Note that $f(x)$ is monotonically decreasing in $T_{p}$. Approximate $f(x)$ around a given point $\tau$ by the tangent line, $f^{\prime}(x)$, passing through $(\tau, f(\tau))$. Solving $f^{\prime}(x)=0$ will give us the approximated solution $T_{p}^{\prime}$ where

$$
\lim _{\tau \rightarrow T_{p}^{*}} T_{p}^{\prime}=T_{p}^{*}
$$

Ideally we want $T_{p}^{\prime}$ to be close to $T_{p}^{*}$. We conducted a numerical study, to confirm this proximity for a wide set of parameter ranges, including cases where $\tau$ differs significantly from $T_{p}^{*}$. Towards this goal, we first randomly sampled different parameter values, and obtained $T_{p}^{*}$ numerically. Next, we calculate the approximated solution $T_{p}^{\prime}$ for the given value of $\tau$.

The numerical study shows that for most of the parameter values, $T_{p}^{\prime}$ performs reasonably well. An example of this is illustrated in Figure 8. In the example, the true solution is $T_{p}^{*}$ is 3 . Varying $\tau$ between 2 and 4 does not lead to big changes in $T_{p}^{\prime}$, whose values remain in the interval $[2.6,3]$. In general, we find that for the parameter settings in our study, varying $\tau$ by $\pm 30 \%$ still yields approximated solutions, $T_{p}^{\prime}$, that are within $10 \%$ of the optimal solution $T_{p}^{*}$. Thus, Taylor approximation indeed provides a good representation of the original problem. 


\section{Figures.}

Buyer
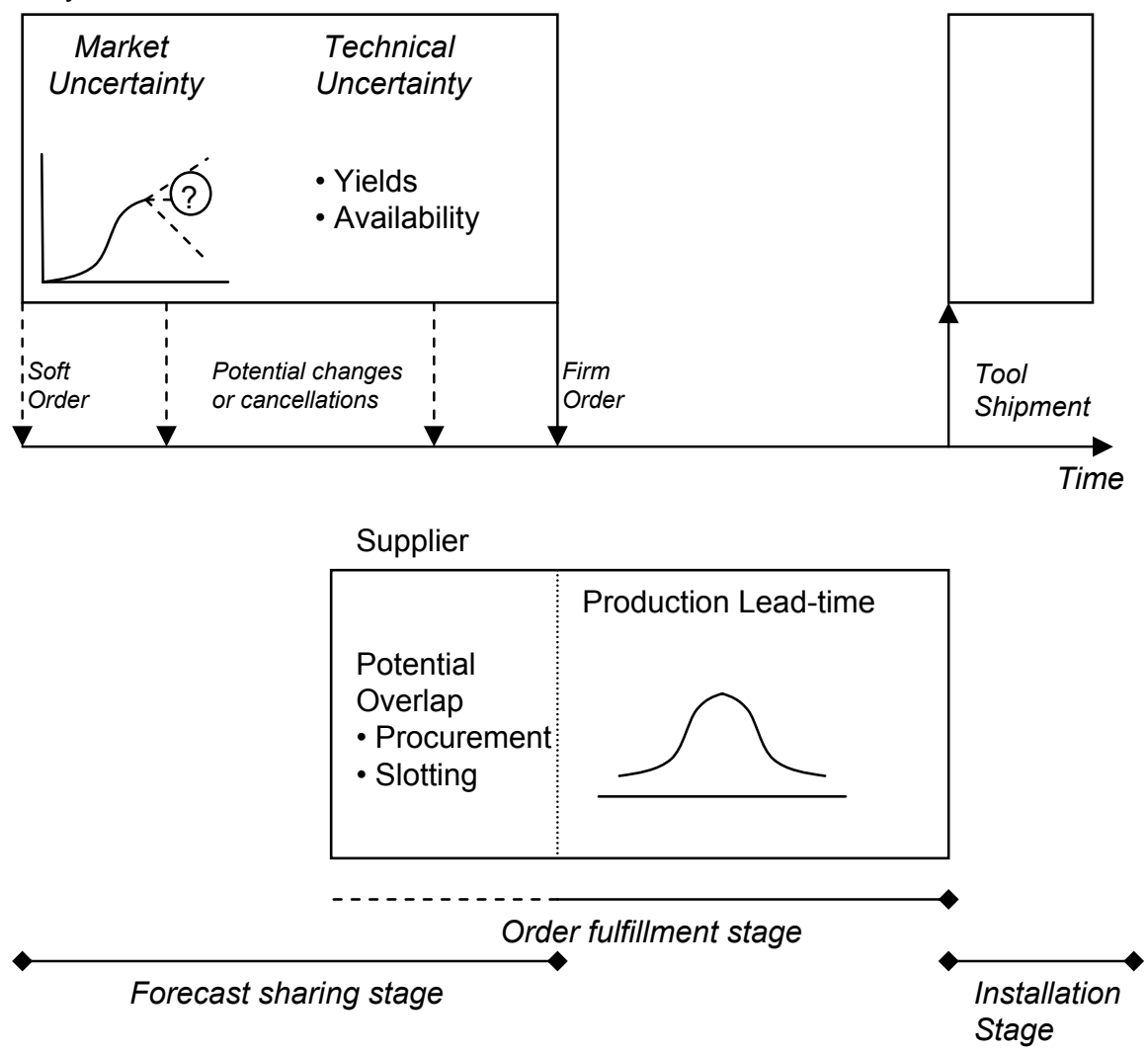

Figure 1: The equipment acquisition cycle

Number of tools (absolute numbers cannot be shown for confidentiality reasons)

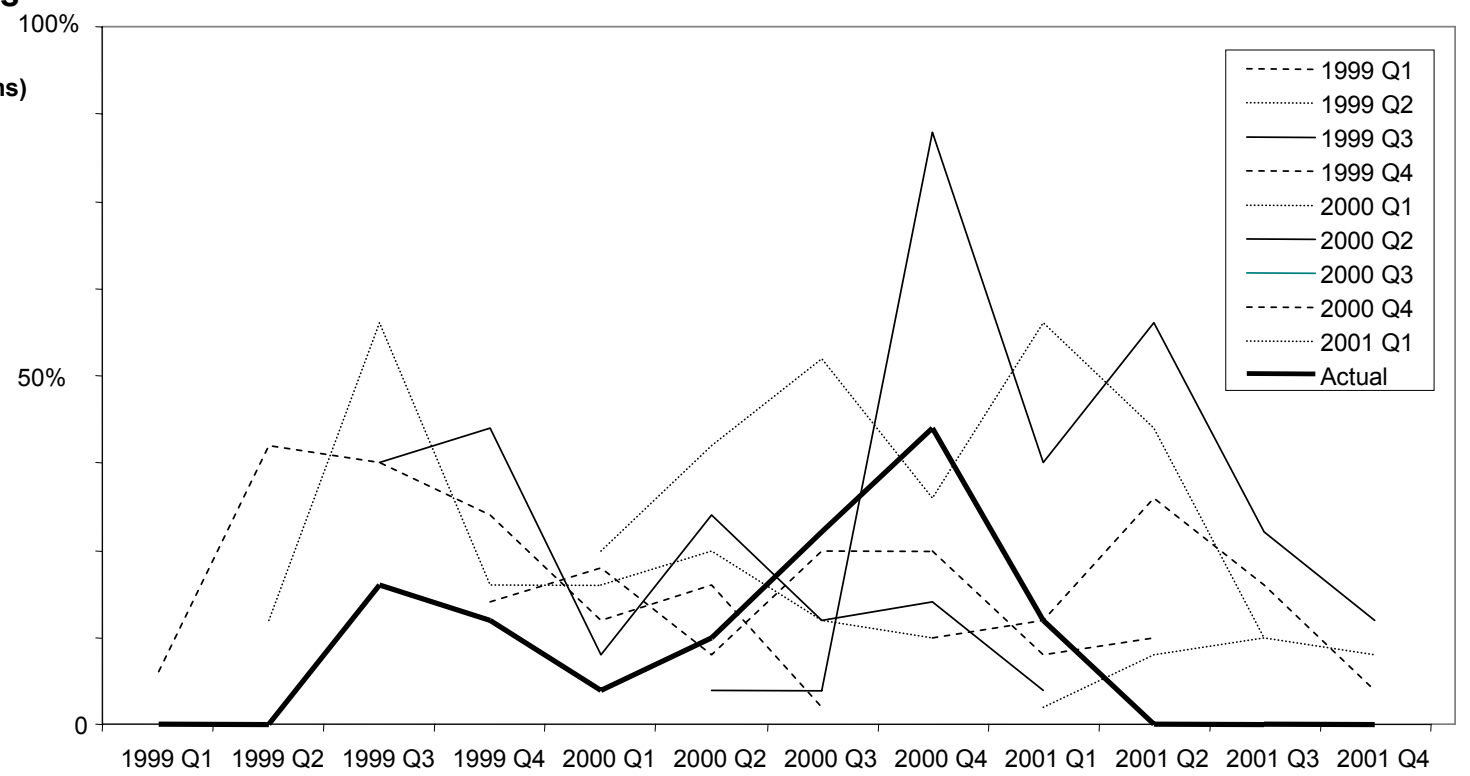

Figure 2: Forecasted (soft) orders versus actual orders 


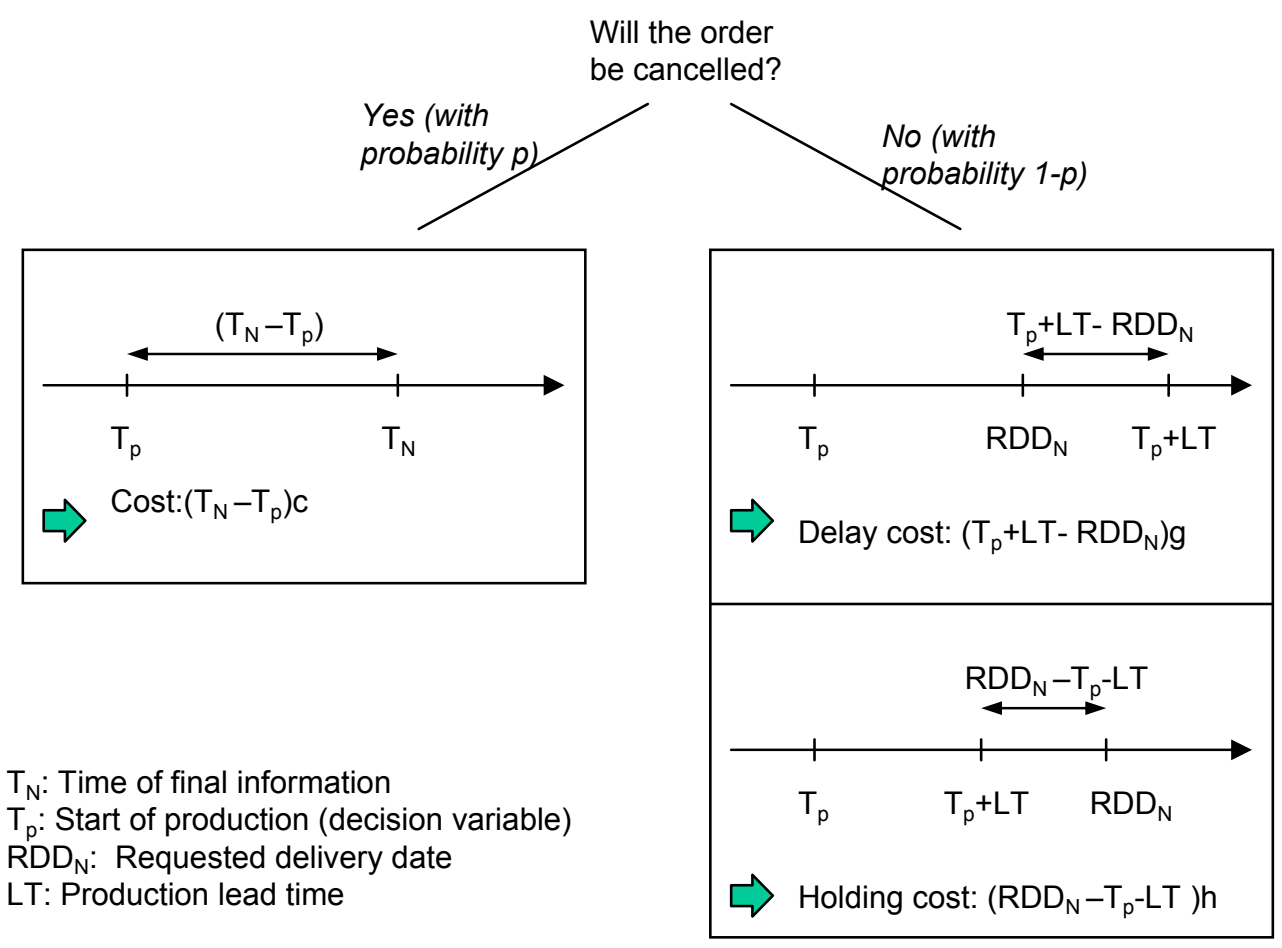

Figure 3: Variable definitions and basic cost drivers

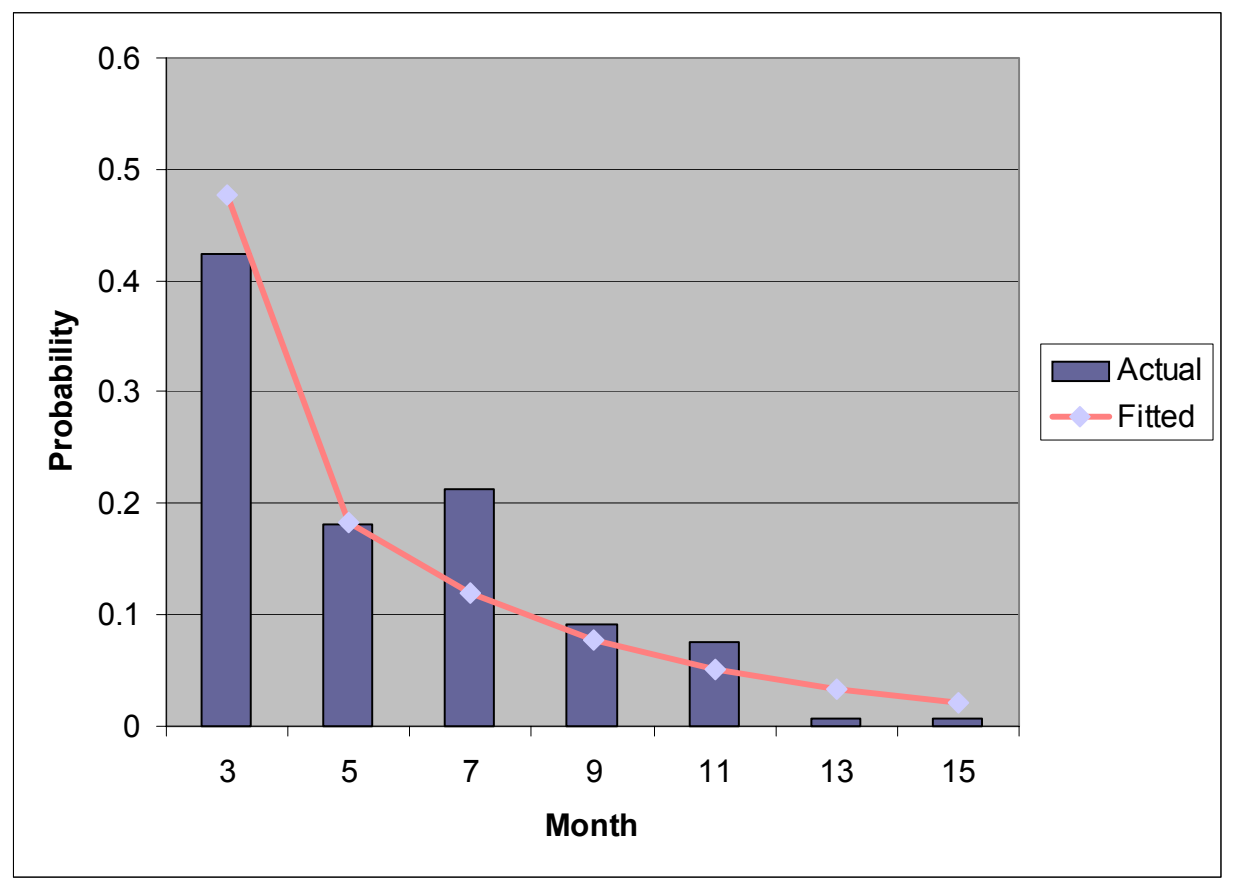

Figure 4: Distribution of G(.) 


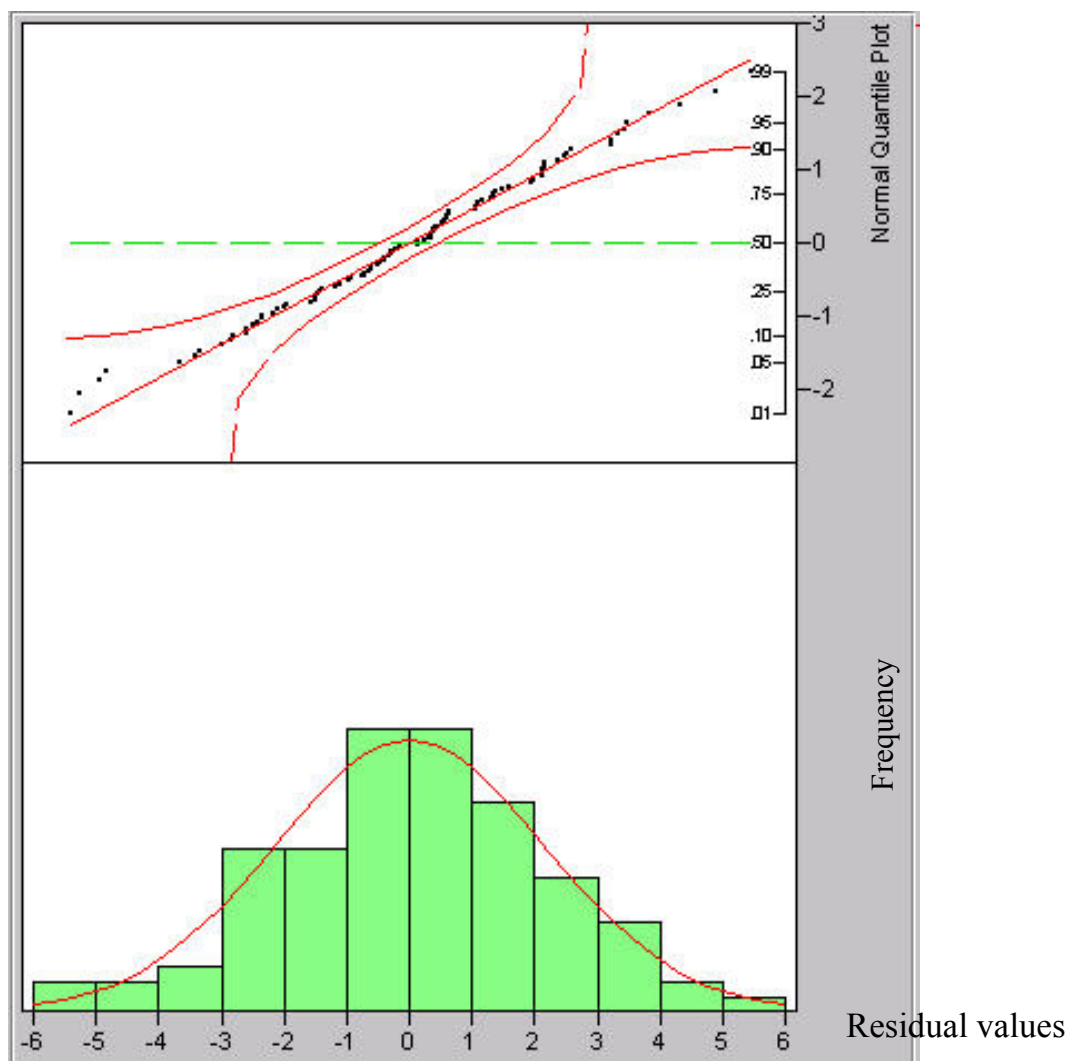

Figure 5: Test for normality

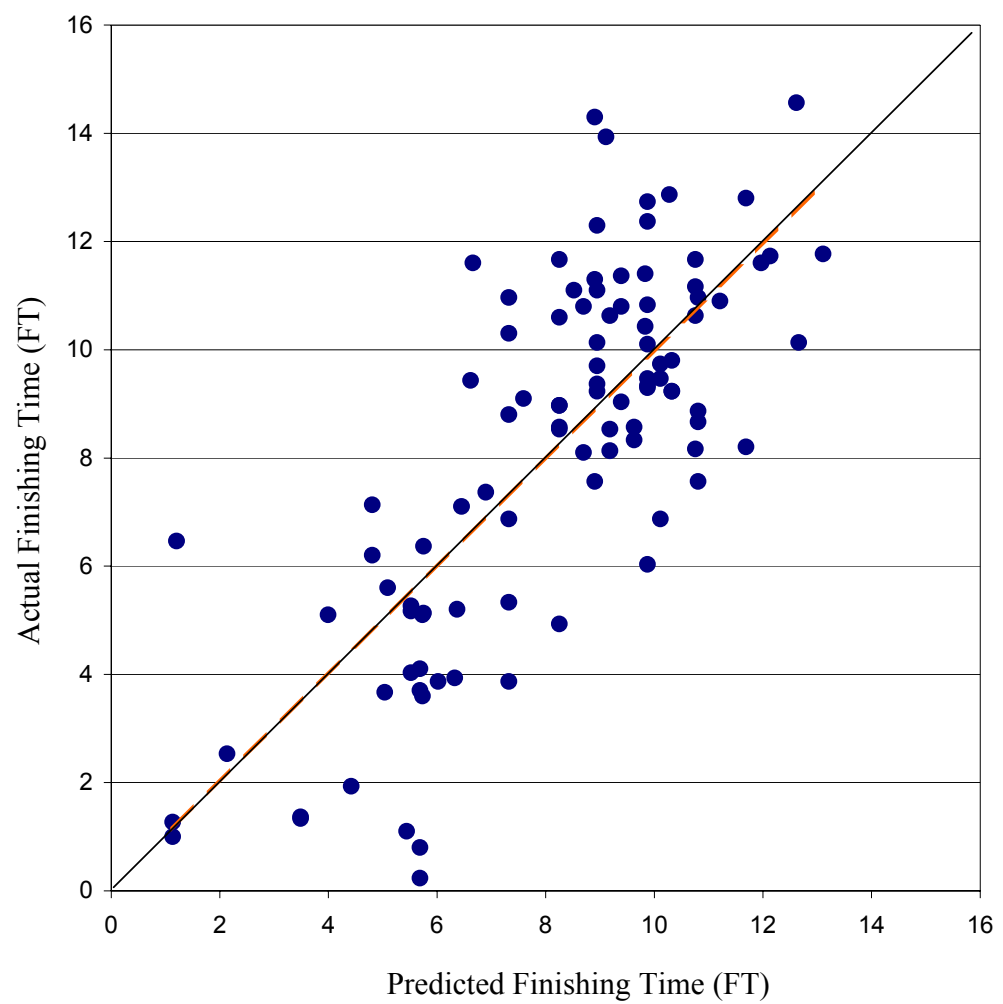

Figure 6: Model Validation 


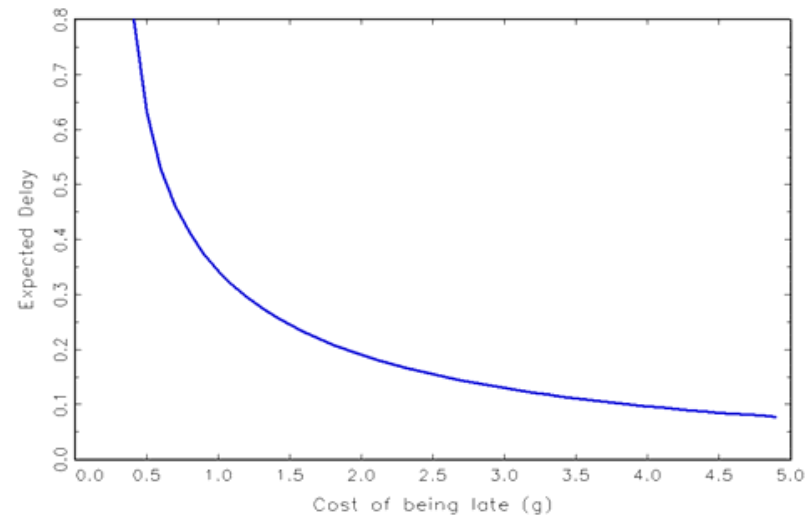

(7-a)

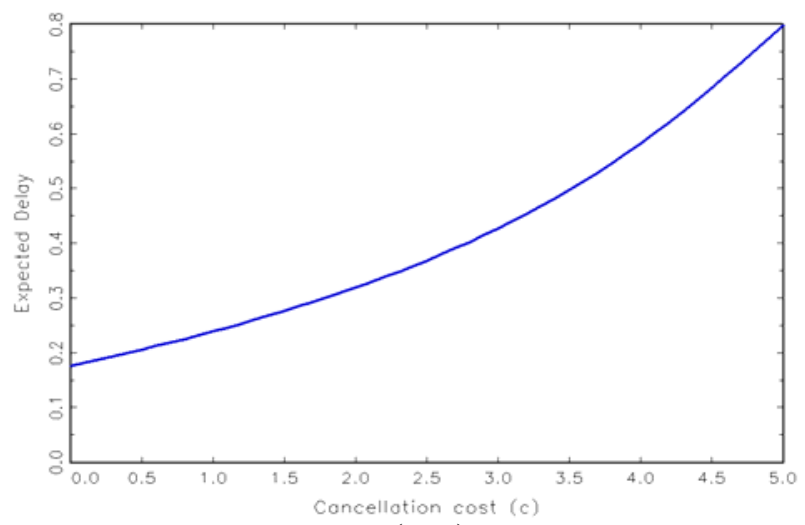

$(7-c)$

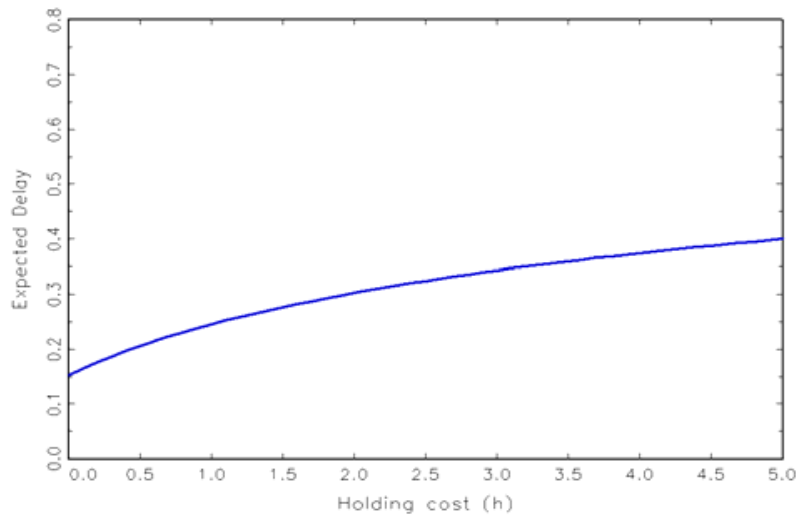

(7-b)

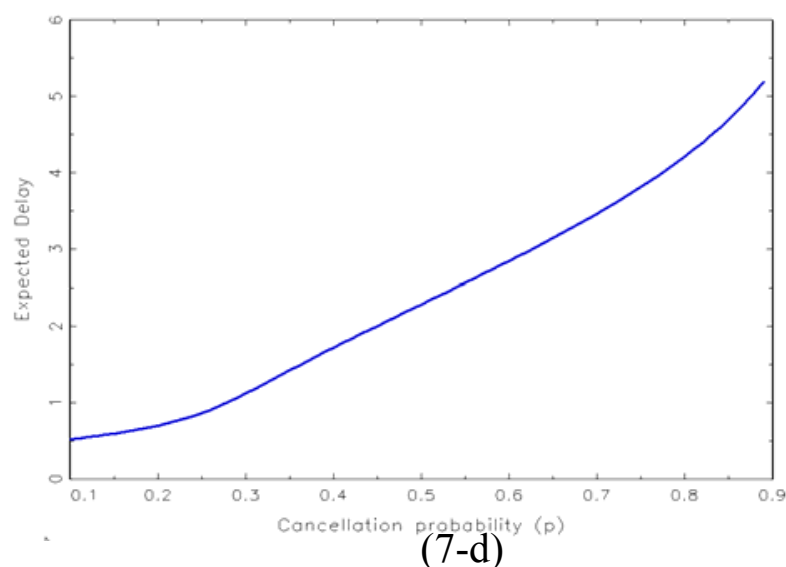

$(7-d)$

Figure 7: Impact of delay cost $g$ (7-a), holding cost $h(7-b)$, cancellation cost $c$ (7-c) and probability $p(7-d)$ on expected delay

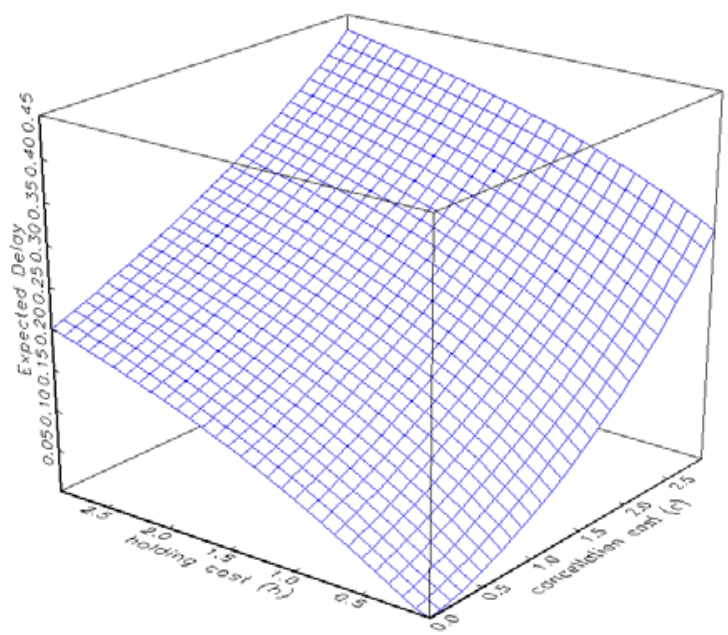

Figure 8 Joint Impact of cancellation cost $c$ and holding cost $h$ on expected delay 


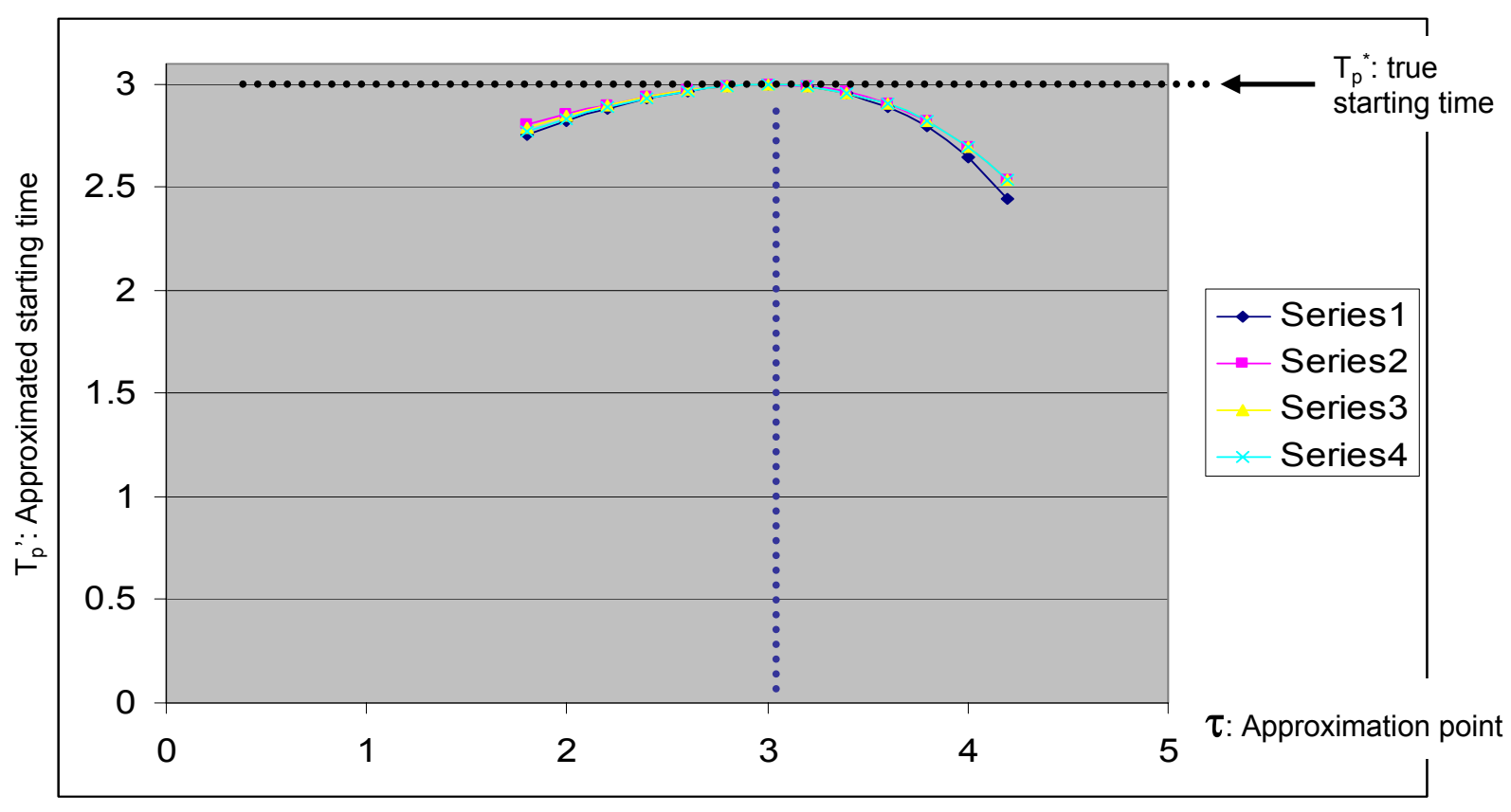

The parameter values for the four series shown above are: $\{\theta 1, \theta 2, \theta 3, \alpha, \beta, \delta\}=\{1,1,0.37,1.1,0.3,0.5\}$, $\{1.5,1,0.37,1.1,0.3,0.5\},\{2,1,0.37,1.1,0.3,0.5\},\{3,1,0.37,1.1,0.3,0.5\}$.

Figure 8 Taylor Approximation

\section{Tables.}

\begin{tabular}{lcccc}
\hline & DEVFAB & NEW & PREMIUM & CHRDD \\
DEVFAB & 1.000 & 0.247 & 0.096 & -0.165 \\
NEW & 0.247 & 1.000 & -0.074 & -0.175 \\
PREMIUM & 0.096 & -0.074 & 1.000 & 0.150 \\
CHRDD & -0.165 & -0.175 & 0.150 & 1.000 \\
\hline
\end{tabular}

Table 1. Correlation Matrix 


\begin{tabular}{|c|c|c|c|c|}
\hline & & \multicolumn{3}{|c|}{ 1-step Estimation } \\
\hline & & Estimate & Std. Err. & $\mathrm{p}$-value \\
\hline \multirow{3}{*}{ 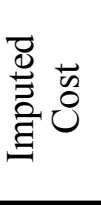 } & $\mathrm{g}$ & 1.000 & $(\mathrm{~N} / \mathrm{A})$ & $(\mathrm{N} / \mathrm{A})$ \\
\hline & $\mathrm{h}$ & 3.031 & 0.013 & 0.002 \\
\hline & $\mathrm{c}$ & 2.108 & 0.003 & 0.010 \\
\hline \multicolumn{2}{|c|}{ Leadtime shift $\delta$} & 0.068 & 0.017 & 0.000 \\
\hline \multicolumn{2}{|c|}{ Standard deviation $\sigma$} & 3.000 & 0.003 & 0.000 \\
\hline \multirow{8}{*}{ 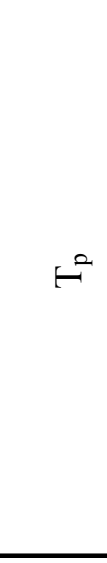 } & Constant $\gamma_{1}$ & -1.085 & 0.005 & 0.000 \\
\hline & Develop $\gamma_{2}$ & -4.326 & 0.005 & 0.000 \\
\hline & New $\gamma_{3}$ & -0.388 & 0.003 & 0.000 \\
\hline & Premium $\gamma_{4}$ & -9.721 & 0.003 & 0.000 \\
\hline & Constant $\rho_{1}$ & 0.827 & 0.004 & 0.000 \\
\hline & Develop $\rho_{2}$ & -2.427 & 0.004 & 0.000 \\
\hline & New $\rho_{3}$ & -0.098 & 0.003 & 0.000 \\
\hline & Premium $\rho_{4}$ & -2.284 & 0.004 & 0.000 \\
\hline \multirow{8}{*}{ 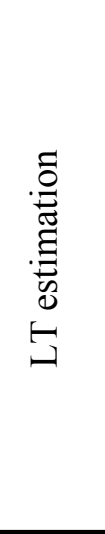 } & Constant $\eta_{1}$ & 4.945 & 0.004 & 0.000 \\
\hline & CHRDD $\eta_{2}$ & 0.966 & 0.004 & 0.000 \\
\hline & Develop $\eta_{3}$ & -3.024 & 0.003 & 0.000 \\
\hline & New $\eta_{4}$ & 0.691 & 0.004 & 0.000 \\
\hline & Premium $\eta_{4}$ & -0.435 & 0.003 & 0.000 \\
\hline & 1HALF98 $\eta_{5}$ & 1.978 & 0.003 & 0.000 \\
\hline & 2 HALF98 $\eta_{6}$ & 1.545 & 0.003 & 0.000 \\
\hline & 1HALF99 $\eta_{7}$ & 1.212 & 0.003 & 0.000 \\
\hline \multicolumn{2}{|c|}{ Correlation coefficient $\lambda$} & 0.207 & 0.003 & 0.000 \\
\hline \multicolumn{2}{|c|}{ Log Likelihood } & & -227.211 & \\
\hline
\end{tabular}

Table 2. Parameter Estimation Results: Full Model 


\begin{tabular}{cccc}
\hline Model & Log-Likelihood & $\chi^{2}$-test statistic & p-value \\
\hline Full Model & -227.211 & & \\
Nested 1: DEVFAB & -232.952 & 11.482 & 0.003 \\
Nested 2: NEW & -234.230 & 14.038 & 0.001 \\
Nested 3: PREMIUM & -233.347 & 12.272 & 0.002 \\
\hline
\end{tabular}

Table 3. Nested Models 